\title{
Type 2 Fuzzy Convexity*
}

\author{
Pishtiwan O. Sabir ${ }^{1}$, Diyar M. Mohammed ${ }^{2}$ \\ ${ }^{1}$ Department of Mathematics, College of Science, University of Sulaimani, Sulaimani, Kurdistan \\ Region, Iraq \\ E-mail: pishtiwan.sabir@univsul.edu.iq \\ ${ }^{2}$ Department of Mathematical Sciences, College of Basic Education, University of Sulaimani, \\ Sulaimani, Kurdistan Region, Iraq
}

\begin{abstract}
In this paper, we introduce a new notion of fuzzy convexity as a generalization of the other types of convexities, and give some connections between type 2 fuzzy convexity and generalized fuzzy convexity. We also discuss the relationship of type 2 fuzzy convex set with generalized strongly convex fuzzy set and semistrongly convex fuzzy set.
\end{abstract}

Keywords: Zadeh's extension principle; Type 2 fuzzy sets; Generalized convex fuzzy sets; Type 2 fuzzy convex sets.

\section{Introduction and Notations}

The concept of fuzzy sets and fuzzy set operations were first introduced by Zadeh [21] in 1965 and subsequently several authors have discussed various aspects of the theory and applications of fuzzy sets [5, 9-10, 24]. Zadeh's work continued research and scholars extensively studied fuzzy convexity such as [1-4, $8,11-13,15-16,18-20]$. In this section, we review the definitions related to the topic and present the notations needed in the rest of the paper. In section two, we give our definitions of generalized strongly convex fuzzy sets, generalized semistrongly convex fuzzy sets and type 2 fuzzy convex sets, and prove some new important theorems and connections among them. The last section contains conclusions related to our main results.

\footnotetext{
* This research first presented in Gen. Math. Notes. Depending on regulations in my University, it is considered as a predatory journal by the college of science at the University of Sulaimani. 
Let $\tilde{A}$ be any fuzzy subset defined on the universal set $X$. Then its grade of membership, denoted by $\mu_{\tilde{A}}$, maps whole members in universal set $X$ to closed interval $[0,1]:=I^{\star}$. If we call fuzzy sets, such as considered, as type 1 fuzzy sets then a type 2 fuzzy set can be defined as a fuzzy set whose membership grade values are type 1 fuzzy sets on $I^{\star}$. A fuzzy set $\tilde{A}$ on $\mathcal{R}$ is quasi-convex if and only if $\mu_{\tilde{A}}(y) \geq \min \left[\mu_{\tilde{A}}\left(y_{1}\right), \mu_{\tilde{A}}\left(y_{2}\right)\right]$, where $y=\left(1-y_{3}\right) y_{2}+y_{3} y_{1}, y_{1}, y_{2} \in \mathcal{R}$ and $y_{3} \in(0,1):=I^{\star \star}$. Frequently, we will denote the family of all fuzzy sets in $X$ by $\mathcal{F}(\mathrm{X})$ and sometimes write $\mu(x, \tilde{A})$ instead of $\mu_{\tilde{A}}(x)$.

Let $\tilde{A}_{i} \in \mathcal{F}(X), i \in I$ ( $I$ is a nonempty index set). Then the standard fuzzy intersection of $\tilde{A}_{i}, \widetilde{\cap}_{i} \tilde{A}_{i}$, is defined by $i n f_{x} \mu\left(x, \tilde{A}_{i}\right)=\Lambda_{x} \mu_{\tilde{A}_{i}}(x)$; the standard fuzzy union of $\tilde{A}_{i}, \widetilde{\mathrm{U}}_{i} \tilde{A}_{i}$, is defined by $\sup _{x} \mu\left(x, \tilde{A}_{i}\right)=\mathrm{V}_{x} \mu_{\tilde{A}_{i}}(x)$; and the complement of $\tilde{A}_{i}, \neg \tilde{A}_{i}$, is defined by $\mu\left(x, \neg \tilde{A}_{i}\right)=1-\mu\left(x, \tilde{A}_{i}\right)$, for all $x \in X$. The union of a fuzzy set $\tilde{A}_{i}$ and its complement $\neg \tilde{A}_{i}$ should not necessarily give the whole universal set $X$. Also, the intersection between the two is not necessarily equal to the empty set.

One of the basic notions of fuzzy subsets is the Zadeh's extension principle. This extension first implied in [21] in an elementary presentation and was finally in [22] and [23] are presented. This principle provides a method for extending crisp mathematical notions to fuzzy quantities as the arguments of the function. Let $g: A_{1} \times A_{2} \times \ldots \times A_{n} \rightarrow B$ given by $y=g\left(a_{1}, a_{2}, \ldots, a_{n}\right)$ and $\tilde{A}_{i} \in \mathcal{F}\left(X_{i}\right)$ for $i=1,2, \ldots n$. Here the set $\tilde{A}=g\left(\tilde{A}_{1}, \tilde{A}_{2}, \ldots, \tilde{A}_{n}\right)$ is defined by

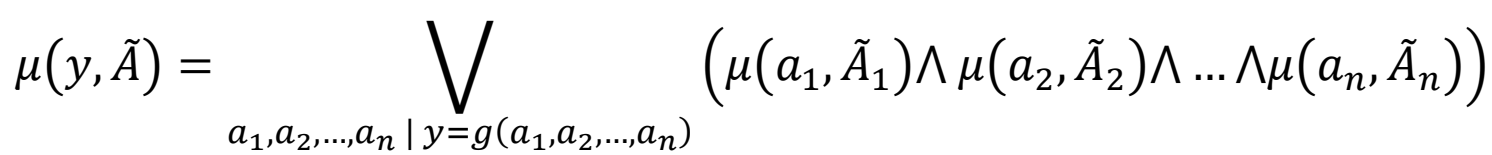

Let $\tilde{A}^{\prime}, \tilde{A}^{\prime \prime} \in \mathcal{F}(X)$. If we denote the extended addition and multiplication by $\bigoplus$ and $\odot$, respectively, then by the Zadeh's principle, one obtains

and

$$
\mu\left(y, \tilde{A}^{\prime} \oplus \tilde{A}^{\prime \prime}\right)=\bigvee_{a_{1}, a_{2} \mid}\left(\mu\left(a_{1}, \tilde{A}^{\prime}\right) \wedge \mu\left(a_{2}, a_{2}, \tilde{A}^{\prime \prime}\right)\right)
$$

$$
\mu\left(y, y_{1} \odot \tilde{A}^{\prime \prime}\right)=\mu\left(y y_{1}^{-1}, \tilde{A}^{\prime \prime}\right), y_{1}>0
$$

As a generalization of Zadeh's fuzzy set, the concept of interval valued fuzzy set was presented by Gorzalczany [6] and introduced for the first time by Turksen 
[17]. Let $\mu(\tilde{A}, x)$ and $\mu(x, \tilde{A})$ denotes for lower fuzzy set and upper fuzzy set about $\mu^{\tilde{A}}$, respectively, then the mapping $\mu_{(x)}^{\tilde{A}}: A \rightarrow[\mu(\tilde{A}, x), \mu(x, \tilde{A})]$ is called an interval valued fuzzy set on $A$ and frequently we shall call generalized fuzzy sets. All generalized fuzzy sets on the universal set $X$ are denoted by $G F S(X)$.

Let $\mu^{\tilde{A}} \in \operatorname{GFS}(X)$ and $\beta_{1}, \beta_{2} \in I^{\star}$. We define ${ }^{+} \mu_{\left[\beta_{1}, \beta_{2}\right]}^{\tilde{A}}$ and $-\mu_{\left(\beta_{1}, \beta_{2}\right)}^{\tilde{A}}$, respectively, as the crisp set of all elements of the universal set $X$ that belongs to lower fuzzy set about $\mu^{\tilde{A}}$ at least to the degree $\beta_{1}$ and upper fuzzy set about $\mu^{\tilde{A}}$ at least to the degree $\beta_{2}$; the ordinary set that contains all elements of the universal set whose membership grades in the given lower set are greater than but do not include the specified value of $\beta_{1}$ and membership grades in the given upper set are greater than but do not include the specified value of $\beta_{2}$. In addition, $\mu^{\tilde{A}}$ is a generalized convex fuzzy set [7], if for any $x_{1}, x_{2} \in \mathcal{R}$ and $\beta \in I^{\star}$, we have $\mu_{\left(\beta x_{1}+(1-\beta) x_{2}\right)}^{\tilde{A}} \geq$ $\mu_{\left(x_{1}\right)}^{\tilde{A}} \wedge \mu_{\left(x_{2}\right)}^{\tilde{A}}$.

\section{Type 2 Fuzzy Convexities}

This section is devotes to present some connections between type 2 fuzzy convex sets and the other types of convexities.

Definition 1: Let $\mu^{\widetilde{\mathcal{F}}} \in G F S(\mathcal{R})$. Then $\mu^{\tilde{\mathcal{F}}}$ is called a generalized strongly convex fuzzy set if

$$
\mu_{(\mu \lambda+(1-\mu) \gamma)}^{\tilde{\mathcal{F}}}>[\mu(\tilde{\mathcal{F}}, \lambda) \wedge \mu(\tilde{\mathcal{F}}, \gamma), \mu(\lambda, \tilde{\mathcal{F}}) \wedge \mu(\gamma, \tilde{\mathcal{F}})]
$$

for every $(\lambda \neq \gamma) \lambda, \gamma \in{ }^{+} \mu_{[0]}^{\tilde{\mathcal{F}}}$ and $\mu \in I^{\star \star}$.

Definition 2: Let $\mu^{\tilde{\mathcal{F}}} \in G F S(\mathcal{R})$. Then $\mu^{\tilde{\mathcal{F}}}$ is called a generalized semistrongly convex fuzzy set if

$$
\mu_{(\mu \lambda+(1-\mu) \gamma)}^{\tilde{\mathcal{F}}}>[\mu(\tilde{\mathcal{F}}, \lambda) \wedge \mu(\tilde{\mathcal{F}}, \gamma), \mu(\lambda, \tilde{\mathcal{F}}) \wedge \mu(\gamma, \tilde{\mathcal{F}})]
$$

for every $\lambda, \gamma \in{ }^{+} \mu_{[0]}^{\tilde{\mathcal{F}}}, \mu(\tilde{\mathcal{F}}, \lambda) \neq \mu(\tilde{\mathcal{F}}, \gamma), \mu(\lambda, \tilde{\mathcal{F}}) \neq \mu(\gamma, \tilde{\mathcal{F}})$ and $\mu \in I^{\star \star}$.

Definition 3: Let $\mu^{\tilde{\mathcal{F}}} \in G F S(\mathcal{R})$. Then $\mu^{\tilde{\mathcal{F}}}$ is called a type 2 fuzzy convex set if 


$$
\begin{aligned}
\mu_{(\mu \lambda+(1-\mu) \gamma)}^{\tilde{\mathcal{F}}}(\beta) & \\
& \geq[\mu(\mu \odot \mu(\tilde{\mathcal{F}}, \lambda) \oplus(1-\mu) \odot \mu(\tilde{\mathcal{F}}, \gamma), \beta), \mu(\beta, \mu \odot \mu(\lambda, \tilde{\mathcal{F}}) \\
& \oplus(1-\mu) \odot \mu(\gamma, \tilde{\mathcal{F}}))]
\end{aligned}
$$

for any $\lambda, \gamma \in{ }^{+} \mu_{[0]}^{\tilde{\mathcal{F}}}, \mu \in I^{\star \star}$ and $\beta \in I^{\star}$.

Theorem 4: Let $\mu^{\widetilde{\mathcal{F}}}, \mu^{\widetilde{\mathcal{H}}} \in G F S(\mathcal{R})$. If both $\mu^{\widetilde{\mathcal{F}}}$ and $\mu^{\widetilde{\mathcal{H}}}$ are type 2 fuzzy convex sets, then $\mu^{\tilde{\mathcal{F}} \widetilde{\tilde{H}}}$ is a type 2 fuzzy convex set.

Proof: Let $\lambda, \gamma \in{ }^{+} \mu_{[0]}^{\tilde{\mathcal{F}} \widetilde{\mathcal{H}}}, \mu \in I^{\star \star}$ and $\beta \in I^{\star}$. By hypothesis, we have

$$
\begin{aligned}
& \mu_{(\mu \lambda+(1-\mu) \gamma)(\beta)}^{\tilde{\mathcal{H}} \widetilde{\mathcal{H}}} \\
& =[\mu(\mu(\tilde{\mathcal{F}} \widetilde{\cap} \widetilde{\mathcal{H}}, \mu \lambda+(1-\mu) \gamma), \beta), \mu(\beta, \mu(\mu \lambda+(1-\mu) \gamma, \widetilde{\mathcal{F}} \widetilde{\cap} \widetilde{\mathcal{H}}))] \\
& =[\mu(\mu(\tilde{\mathcal{F}}, \mu \lambda+(1-\mu) \gamma) \wedge \mu(\widetilde{\mathcal{H}}, \mu \lambda+(1-\mu) \gamma), \beta), \mu(\beta, \mu(\mu \lambda+(1-\mu) \gamma, \tilde{\mathcal{F}}) \\
& \wedge \mu(\mu \lambda+(1-\mu) \gamma, \widetilde{\mathcal{H}}))] \\
& =[\mu(\mu(\tilde{\mathcal{F}}, \mu \lambda+(1-\mu) \gamma), \beta) \\
& \wedge \mu(\mu(\widetilde{\mathcal{H}}, \mu \lambda+(1-\mu) \gamma), \beta), \mu(\beta, \mu(\mu \lambda+(1-\mu) \gamma, \tilde{\mathcal{F}})) \\
& \wedge \mu(\beta, \mu(\mu \lambda+(1-\mu) \gamma, \widetilde{\mathcal{H}}))] \\
& =[\mu(\mu(\tilde{\mathcal{F}}, \mu \lambda+(1-\mu) \gamma), \beta), \mu(\beta, \mu(\mu \lambda+(1-\mu) \gamma, \tilde{\mathcal{F}}))] \\
& \wedge[\mu(\mu(\widetilde{\mathcal{H}}, \mu \lambda+(1-\mu) \gamma), \beta), \mu(\beta, \mu(\mu \lambda+(1-\mu) \gamma, \widetilde{\mathcal{H}}))]
\end{aligned}
$$

Since $\mu^{\tilde{\mathcal{F}}}$ and $\mu^{\widetilde{\mathcal{H}}}$ are type 2 fuzzy convex sets, we get

$$
\begin{aligned}
\mu_{(\mu \lambda+(1-\mu) \gamma)}^{\tilde{\mathcal{F}} \widetilde{\mathcal{H}}}(\beta) & \\
& \geq[\mu(\mu \odot \mu(\tilde{\mathcal{F}}, \lambda) \oplus(1-\mu) \odot \mu(\tilde{\mathcal{F}}, \gamma), \beta), \mu(\beta, \mu \odot \mu(\lambda, \tilde{\mathcal{F}}) \\
& \oplus(1-\mu) \odot \mu(\gamma, \widetilde{\mathcal{F}}))] \\
& \wedge[\mu(\mu \odot \mu(\widetilde{\mathcal{H}}, \lambda) \oplus(1-\mu) \odot \mu(\widetilde{\mathcal{H}}, \gamma), \beta), \mu(\beta, \mu \odot \mu(\lambda, \widetilde{\mathcal{H}}) \\
& \oplus(1-\mu) \odot \mu(\gamma, \widetilde{\mathcal{H}}))]
\end{aligned}
$$


Finally, from the Zadeh's extension principle we obtain

$$
\begin{aligned}
& \mu_{(\mu \lambda+(1-\mu) \gamma)(\beta)}^{\tilde{\mathcal{F}} \widetilde{\mathcal{H}}} \\
& \geq \bigvee_{\beta_{1}, \beta_{2} \mid \beta=\beta_{1}+\beta_{2}}\left[\mu\left(\mu \odot \mu(\tilde{\mathcal{F}}, \lambda), \beta_{1}\right)\right. \\
& \wedge \mu\left((1-\mu) \odot \mu(\tilde{\mathcal{F}}, \gamma), \beta_{2}\right), \mu\left(\beta_{1}, \mu \odot \mu(\lambda, \tilde{\mathcal{F}})\right) \\
& \left.\wedge \mu\left(\beta_{2},(1-\mu) \odot \mu(\gamma, \tilde{\mathcal{F}})\right)\right] \\
& \wedge \quad \bigvee\left[\mu\left(\mu \odot \mu(\widetilde{\mathcal{H}}, \lambda), \beta_{1}\right)\right. \\
& \beta_{1}, \beta_{2} \mid \beta=\beta_{1}+\beta_{2} \\
& \wedge \mu\left((1-\mu) \odot \mu(\widetilde{\mathcal{H}}, \gamma), \beta_{2}\right), \mu\left(\beta_{1}, \mu \odot \mu(\lambda, \widetilde{\mathcal{H}})\right) \\
& \left.\wedge \mu\left(\beta_{2},(1-\mu) \odot \mu(\gamma, \tilde{\mathcal{H}})\right)\right] \\
& =\bigvee_{\beta_{1}, \beta_{2} \mid \beta=\beta_{1}+\beta_{2}}\left[\mu\left(\mu(\tilde{\mathcal{F}}, \lambda), \mu^{-1} \beta_{1}\right) \wedge \mu\left(\mu(\tilde{\mathcal{F}}, \gamma),(1-\mu)^{-1} \beta_{2}\right), \mu\left(\mu^{-1} \beta_{1}, \mu(\lambda, \tilde{\mathcal{F}})\right)\right. \\
& \left.\wedge \mu\left((1-\mu)^{-1} \beta_{2}, \mu(\gamma, \widetilde{\mathcal{F}})\right)\right] \\
& \wedge\left[\mu\left(\mu(\widetilde{\mathcal{H}}, \lambda), \mu^{-1} \beta_{1}\right)\right. \\
& \wedge \mu\left(\mu(\widetilde{\mathcal{H}}, \gamma),(1-\mu)^{-1} \beta_{2}\right), \mu\left(\mu^{-1} \beta_{1}, \mu(\lambda, \widetilde{\mathcal{H}})\right) \\
& \left.\wedge \mu\left((1-\mu)^{-1} \beta_{2}, \mu(\gamma, \tilde{\mathcal{H}})\right)\right] \\
& =\bigvee_{\beta_{1}, \beta_{2} \mid \beta=\mu \beta_{1}+(1-\mu) \beta_{2}}\left[\mu\left(\mu(\tilde{\mathcal{F}}, \lambda), \beta_{1}\right) \wedge \mu\left(\mu(\tilde{\mathcal{F}}, \gamma), \beta_{2}\right), \mu\left(\beta_{1}, \mu(\lambda, \tilde{\mathcal{F}})\right)\right. \\
& \left.\wedge \mu\left(\beta_{2}, \mu(\gamma, \tilde{\mathcal{F}})\right)\right] \\
& \wedge\left[\mu\left(\mu(\widetilde{\mathcal{H}}, \lambda), \beta_{1}\right) \wedge \mu\left(\mu(\widetilde{\mathcal{H}}, \gamma), \beta_{2}\right), \mu\left(\beta_{1}, \mu(\lambda, \widetilde{\mathcal{H}})\right)\right. \\
& \left.\wedge \mu\left(\beta_{2}, \mu(\gamma, \widetilde{\mathcal{H}})\right)\right] \\
& =\bigvee_{\beta_{1}, \beta_{2} \mid \beta=\mu \beta_{1}+(1-\mu) \beta_{2}}\left[\mu\left(\mu(\tilde{\mathcal{F}}, \lambda), \beta_{1}\right) \wedge \mu\left(\mu(\tilde{\mathcal{F}}, \gamma), \beta_{2}\right) \wedge \mu\left(\mu(\widetilde{\mathcal{H}}, \lambda), \beta_{1}\right)\right. \\
& \wedge \mu\left(\mu(\widetilde{\mathcal{H}}, \gamma), \beta_{2}\right), \mu\left(\beta_{1}, \mu(\lambda, \tilde{\mathcal{F}})\right) \wedge \mu\left(\beta_{2}, \mu(\gamma, \tilde{\mathcal{F}})\right) \wedge \mu\left(\beta_{1}, \mu(\lambda, \widetilde{\mathcal{H}})\right) \\
& \left.\wedge \mu\left(\beta_{2}, \mu(\gamma, \widetilde{\mathcal{H}})\right)\right]
\end{aligned}
$$




$$
\begin{aligned}
& =\bigvee_{\beta_{1}, \beta_{2} \mid \beta=\mu \beta_{1}+(1-\mu) \beta_{2}}\left[\mu\left(\mu(\tilde{\mathcal{F}} \widetilde{\cap} \widetilde{\mathcal{H}}, \lambda), \beta_{1}\right)\right. \\
& \left.\wedge \mu\left(\mu(\tilde{\mathcal{F}} \widetilde{\cap} \widetilde{\mathcal{H}}, \gamma), \beta_{2}\right), \mu\left(\beta_{1}, \mu(\lambda, \tilde{\mathcal{F}} \widetilde{\cap} \widetilde{\mathcal{H}})\right) \wedge \mu\left(\beta_{2}, \mu(\gamma, \tilde{\mathcal{F}} \widetilde{\cap} \widetilde{\mathcal{H}})\right)\right] \\
& =\bigvee_{\beta_{1}, \beta_{2} \mid \beta=\beta_{1}+\beta_{2}}\left[\mu\left(\mu(\tilde{\mathcal{F}} \widetilde{\cap} \widetilde{\mathcal{H}}, \lambda), \mu^{-1} \beta_{1}\right)\right. \\
& \wedge \mu\left(\mu(\tilde{\mathcal{F}} \widetilde{\cap} \widetilde{\mathcal{H}}, \gamma),(1-\mu)^{-1} \beta_{2}\right), \mu\left(\mu^{-1} \beta_{1}, \mu(\lambda, \tilde{\mathcal{F}} \widetilde{\cap} \widetilde{\mathcal{H}})\right) \\
& \left.\wedge \mu\left((1-\mu)^{-1} \beta_{2}, \mu(\gamma, \tilde{\mathcal{F}} \widetilde{\cap} \widetilde{\mathcal{H}})\right)\right] \\
& =\bigvee_{\beta_{1}, \beta_{2} \mid \beta=\beta_{1}+\beta_{2}}\left[\mu\left(\mu \odot \mu(\tilde{\mathcal{F}} \widetilde{\cap} \tilde{\mathcal{H}}, \lambda), \beta_{1}\right)\right. \\
& \wedge \mu\left((1-\mu) \odot \mu(\tilde{\mathcal{F}} \widetilde{\cap} \widetilde{\mathcal{H}}, \gamma), \beta_{2}\right), \mu\left(\beta_{1}, \mu \odot \mu(\lambda, \tilde{\mathcal{F}} \widetilde{\cap} \widetilde{\mathcal{H}})\right) \\
& \left.\wedge \mu\left(\beta_{2}, \mu \odot \mu(\gamma, \tilde{\mathcal{F}} \widetilde{\cap} \widetilde{\mathcal{H}})\right)\right] \\
& =[\mu(\mu \odot \mu(\tilde{\mathcal{F}} \widetilde{\cap} \widetilde{\mathcal{H}}, \lambda) \oplus(1-\mu) \odot \mu(\tilde{\mathcal{F}} \widetilde{\cap} \widetilde{\mathcal{H}}, \gamma), \beta), \mu(\beta, \mu \odot \mu(\lambda, \tilde{\mathcal{F}} \widetilde{\cap} \widetilde{\mathcal{H}}) \\
& \oplus(1-\mu) \odot \mu(\gamma, \tilde{\mathcal{F}} \widetilde{\cap} \widetilde{\mathcal{H}}))]
\end{aligned}
$$

Remark 5: Let $\mu^{\tilde{\mathcal{F}}}, \mu^{\widetilde{\mathcal{H}}} \in G F S(\mathcal{R})$. If $\mu^{\tilde{\mathcal{F}}}$ and $\mu^{\widetilde{\mathcal{H}}}$ are type 2 fuzzy convex sets, then standard fuzzy union of $\mu^{\tilde{\mathcal{F}}}$ and $\mu^{\widetilde{\mathcal{H}}}$ is not necessarily a type 2 fuzzy convex set. It is easy to see that for $\lambda=0.5=\mu, \gamma=0.7$ and $\beta=1$, the following example covers the remark;

$$
\begin{gathered}
\mu(\gamma, \mu(\lambda, \widetilde{\mathcal{H}}))= \begin{cases}(\gamma-\lambda) /(1-\lambda) & \text { if } \gamma \in[\lambda, 1] \\
\gamma^{2} & \text { if } \gamma \in(0, \lambda)\end{cases} \\
\mu(\mu(\widetilde{\mathcal{F}}, \lambda), \gamma)= \begin{cases}(1-\gamma) / 0.5 & \text { if } \gamma \in(0.5,1] \\
1.0 & \text { if } \gamma \in[\lambda, 0.5] \\
\gamma / \lambda & \text { if } \gamma \in[0, \lambda)\end{cases} \\
\mu(\mu(\widetilde{\mathcal{H}}, \lambda), \gamma)= \begin{cases}\gamma \lambda & \text { if } \gamma \in(\lambda, 1) \\
(\lambda-\gamma) / \lambda & \text { if } \gamma \in[0, \lambda]\end{cases}
\end{gathered}
$$




$$
\mu(\gamma, \mu(\lambda, \tilde{\mathcal{F}}))= \begin{cases}1.0 & \text { if } \gamma=1 \\ (1-\gamma) /(1-\sqrt{\lambda}) & \text { if } \gamma \in(\sqrt{\lambda}, 1) \\ (\gamma-\lambda) /(\sqrt{\lambda}-\lambda) & \text { if } \gamma \in(\lambda, \sqrt{\lambda}] \\ 0.0 & \text { if } \gamma \in[0, \lambda]\end{cases}
$$

Theorem 6: Let $\mu^{\tilde{\mathcal{F}}} \in G F S(\mathcal{R})$. If for all $\lambda, \gamma \in{ }^{+} \mu_{[0]}^{\tilde{\mathcal{F}}}, v>0, \mu_{(\nu \lambda)}^{\tilde{\mathcal{F}}}+\mu_{(\nu \gamma)}^{\tilde{\mathcal{F}}} \subseteq$ $v \mu_{(\lambda+\gamma)}^{\tilde{\mathcal{F}}}$, then $\mu^{\tilde{\mathcal{F}}}$ is a type 2 fuzzy convex set.

Proof: Let $\mu \in I^{\star \star}$ and $\beta \in I^{\star}$. From the definition of generalized fuzzy sets and hypothesis we have,

$$
\begin{aligned}
& \mu_{(\mu \lambda+(1-\mu) \gamma)(\beta)}^{\tilde{\mathcal{F}}}=[\mu(\mu(\tilde{\mathcal{F}}, \mu \lambda+(1-\mu) \gamma), \beta), \mu(\beta, \mu(\mu \lambda+(1-\mu) \gamma, \tilde{\mathcal{F}}))] \\
& \geq[\mu(\mu(\tilde{\mathcal{F}}, \mu \lambda) \oplus \mu(\tilde{\mathcal{F}},(1-\mu) \gamma), \beta), \mu(\beta, \mu(\mu \lambda, \tilde{\mathcal{F}}) \oplus \mu((1-\mu) \gamma, \tilde{\mathcal{F}}))]
\end{aligned}
$$

From the Zadeh's extension principle we get

$$
\begin{aligned}
& \mu_{(\mu \lambda+(1-\mu) \gamma)(\beta)}^{\tilde{\mathcal{F}}} \geq \bigvee_{\beta_{1}, \beta_{2} \mid \beta=\beta_{1}+\beta_{2}}\left[\mu\left(\mu(\tilde{\mathcal{F}}, \mu \lambda), \beta_{1}\right)\right. \\
& \left.\wedge \mu\left(\mu(\tilde{\mathcal{F}},(1-\mu) \gamma), \beta_{2}\right), \mu\left(\beta_{1}, \mu(\mu \lambda, \tilde{\mathcal{F}})\right) \wedge \mu\left(\beta_{2}, \mu((1-\mu) \gamma, \tilde{\mathcal{F}})\right)\right] \\
& =\bigvee_{\beta_{1}, \beta_{2} \mid \beta=\mu \beta_{1}+(1-\mu) \beta_{2}}^{\wedge \mu\left(\mu(\tilde{\mathcal{F}},(1-\mu) \gamma),(1-\mu) \beta_{2}\right), \mu\left(\mu \beta_{1}, \mu(\mu \lambda, \tilde{\mathcal{F}})\right)} \\
& \left.\wedge \mu\left((1-\mu) \beta_{2}, \mu((1-\mu) \gamma, \tilde{\mathcal{F}})\right)\right]
\end{aligned}
$$

On the other hand, the hypothesis of the theorem gives that

$$
\begin{aligned}
\mu\left(\mu(\tilde{\mathcal{F}}, \mu \lambda), \mu \beta_{1}\right) \wedge \mu\left(\mu(\tilde{\mathcal{F}},(1-\mu) \gamma),(1-\mu) \beta_{2}\right) \\
=\mu\left(\mu \odot \mu(\tilde{\mathcal{F}}, \lambda), \mu \beta_{1}\right) \wedge \mu\left((1-\mu) \odot \mu(\tilde{\mathcal{F}}, \gamma),(1-\mu) \beta_{2}\right)
\end{aligned}
$$

and 


$$
\begin{aligned}
\mu\left(\mu \beta_{1}, \mu(\mu \lambda, \tilde{\mathcal{F}})\right) \wedge \mu\left((1-\mu) \beta_{2}, \mu((1-\mu) \gamma, \tilde{\mathcal{F}})\right) \\
\quad=\mu\left(\mu \beta_{1}, \mu \odot \mu(\lambda, \tilde{\mathcal{F}})\right) \wedge \mu\left((1-\mu) \beta_{2},(1-\mu) \odot \mu(\gamma, \tilde{\mathcal{F}})\right)
\end{aligned}
$$

Therefore,

$$
\begin{aligned}
\mu_{(\mu \lambda+(1-\mu) \gamma)(\beta)}^{\tilde{\mathcal{F}}} & \geq \bigvee_{\beta_{1}, \beta_{2} \mid \beta=\mu \beta_{1}+(1-\mu) \beta_{2}}\left[\mu\left(\mu \odot \mu(\tilde{\mathcal{F}}, \lambda), \mu \beta_{1}\right)\right. \\
& \wedge \mu\left((1-\mu) \odot \mu(\tilde{\mathcal{F}}, \gamma),(1-\mu) \beta_{2}\right), \mu\left(\mu \beta_{1}, \mu \odot \mu(\lambda, \tilde{\mathcal{F}})\right) \\
& \left.\wedge \mu\left((1-\mu) \beta_{2},(1-\mu) \odot \mu(\gamma, \tilde{\mathcal{F}})\right)\right]
\end{aligned}
$$

Again from the extension principle for fuzzy sets, we obtain

$$
\begin{aligned}
& \mu_{(\mu \lambda+(1-\mu) \gamma)(\beta)}^{\tilde{\mathcal{F}}} \\
& \geq \bigvee_{\beta_{1}, \beta_{2} \mid \beta=\mu \beta_{1}+(1-\mu) \beta_{2}}\left[\mu\left(\mu(\tilde{\mathcal{F}}, \lambda), \beta_{1}\right)\right. \\
& \wedge \mu\left(\mu(\tilde{\mathcal{F}}, \gamma),(1-\mu)^{-1}(1-\mu) \beta_{2}\right), \mu\left(\beta_{1}, \mu(\lambda, \tilde{\mathcal{F}})\right) \\
& \left.\wedge \mu\left(\beta_{2}, \mu\left((1-\mu)(1-\mu)^{-1} \gamma, \tilde{\mathcal{F}}\right)\right)\right] \\
& =\bigvee_{\beta_{1}, \beta_{2} \mid \beta=\mu \beta_{1}+(1-\mu) \beta_{2}}\left[\mu\left(\mu(\tilde{\mathcal{F}}, \lambda), \beta_{1}\right) \wedge \mu\left(\mu(\tilde{\mathcal{F}}, \gamma), \beta_{2}\right), \mu\left(\beta_{1}, \mu(\lambda, \tilde{\mathcal{F}})\right)\right. \\
& \left.\wedge \mu\left(\beta_{2}, \mu(\gamma, \tilde{\mathcal{F}})\right)\right] \\
& =\bigvee_{\beta_{1}, \beta_{2} \mid \beta=\beta_{1}+\beta_{2}}\left[\mu\left(\mu(\tilde{\mathcal{F}}, \lambda), \mu^{-1} \beta_{1}\right) \wedge \mu\left(\mu(\tilde{\mathcal{F}}, \gamma),(1-\mu)^{-1} \beta_{2}\right), \mu\left(\mu^{-1} \beta_{1}, \mu(\lambda, \tilde{\mathcal{F}})\right)\right. \\
& \left.\wedge \mu\left((1-\mu)^{-1} \beta_{2}, \mu(\gamma, \tilde{\mathcal{F}})\right)\right] \\
& =\bigvee_{\beta_{1}, \beta_{2} \mid \beta=\beta_{1}+\beta_{2}}\left[\mu\left(\mu \odot \mu(\tilde{\mathcal{F}}, \lambda), \beta_{1}\right)\right. \\
& \wedge \mu\left((1-\mu) \odot \mu(\tilde{\mathcal{F}}, \gamma), \beta_{2}\right), \mu\left(\beta_{1}, \mu \odot \mu(\lambda, \tilde{\mathcal{F}})\right) \\
& \left.\wedge \mu\left(\beta_{2},(1-\mu) \odot \mu(\gamma, \tilde{\mathcal{F}})\right)\right]
\end{aligned}
$$




$$
\begin{gathered}
=[\mu(\mu \odot \mu(\tilde{\mathcal{F}}, \lambda) \oplus(1-\mu) \odot \mu(\tilde{\mathcal{F}}, \gamma), \beta), \mu(\beta, \mu \odot \mu(\lambda, \tilde{\mathcal{F}}) \oplus(1-\mu) \\
\odot \mu(\gamma, \tilde{\mathcal{F}}))]
\end{gathered}
$$

Theorem 7: Let $\mu^{\tilde{\mathcal{F}}} \in G F S(\mathcal{R})$. If $\mu^{\tilde{\mathcal{F}}}$ is a type 2 fuzzy convex set then $\mu_{(v)}^{\tilde{\mathcal{F}}}$ is a generalized convex fuzzy set in $I^{\star}$ for all $v \in{ }^{+} \mu_{[0]}^{\tilde{\mathcal{F}}}$.

Proof: For $(\lambda, \gamma) \in I^{\star} \times I^{\star}$ and $\mu \in I^{\star \star}$. The definition of type 2 fuzzy convex set gives that,

$$
\begin{aligned}
\mu_{(\mu \nu+(1-\mu) v)}^{\tilde{\mathcal{F}}} & (\mu \lambda+(1-\mu) \gamma) \\
& \geq[\mu(\mu \odot \mu(\tilde{\mathcal{F}}, v) \oplus(1-\mu) \odot \mu(\tilde{\mathcal{F}}, v), \mu \lambda+(1-\mu) \gamma), \mu(\mu \lambda \\
& +(1-\mu) \gamma, \mu \odot \mu(v, \tilde{\mathcal{F}}) \oplus(1-\mu) \odot \mu(\nu, \tilde{\mathcal{F}}))]
\end{aligned}
$$

Therefore, the Zadeh's principle implies that,

$$
\begin{aligned}
& \mu_{(\mu \nu+(1-\mu) v)(\mu \lambda+(1-\mu) \gamma)}^{\tilde{\mathcal{F}}} \\
& \geq \bigvee_{\beta_{1}, \beta_{2} \mid \mu \lambda+(1-\mu) \gamma=\beta_{1}+\beta_{2}}\left[\mu\left(\mu \odot \mu(\tilde{\mathcal{F}}, v), \beta_{1}\right)\right. \\
& \Lambda \mu\left((1-\mu) \odot \mu(\tilde{\mathcal{F}}, v), \beta_{2}\right), \mu\left(\beta_{1}, \mu \odot \mu(\nu, \tilde{\mathcal{F}})\right) \\
& \left.\wedge \mu\left(\beta_{2},(1-\mu) \odot \mu(\nu, \tilde{\mathcal{F}})\right)\right] \\
& =\bigvee_{\beta_{1}, \beta_{2} \mid \mu \lambda+(1-\mu) \gamma=\beta_{1}+\beta_{2}}\left[\mu\left(\mu(\tilde{\mathcal{F}}, v), \mu^{-1} \beta_{1}\right)\right. \\
& \wedge \mu\left(\mu(\tilde{\mathcal{F}}, v),(1-\mu)^{-1} \beta_{2}\right), \mu\left(\mu^{-1} \beta_{1}, \mu(\nu, \tilde{\mathcal{F}})\right) \\
& \left.\wedge \mu\left((1-\mu)^{-1} \beta_{2}, \mu(\nu, \tilde{\mathcal{F}})\right)\right] \\
& \begin{array}{l}
=\bigvee_{\beta_{1}, \beta_{2} \mid \mu \lambda+(1-\mu) \gamma=\mu \beta_{1}+(1-\mu) \beta_{2}}\left[\mu\left(\mu(\tilde{\mathcal{F}}, v), \beta_{1}\right) \wedge \mu\left(\mu(\tilde{\mathcal{F}}, v), \beta_{2}\right), \mu\left(\beta_{1}, \mu(v, \tilde{\mathcal{F}})\right)\right. \\
\left.\wedge \mu\left(\beta_{2}, \mu(v, \tilde{\mathcal{F}})\right)\right]
\end{array}
\end{aligned}
$$

On the other hand, for any $\left(\beta_{1}, \beta_{2}\right) \in I^{\star 2}$ and $\mu \in I^{\star \star}$ the equality $\mu \beta_{1}+$ $(1-\mu) \beta_{2}=\mu \lambda+(1-\mu) \gamma$ gives that 


$$
\begin{aligned}
{\left[\mu\left(\mu(\tilde{\mathcal{F}}, v), \beta_{1}\right) \wedge \mu\left(\mu(\tilde{\mathcal{F}}, v), \beta_{2}\right), \mu\left(\beta_{1}, \mu(v, \tilde{\mathcal{F}})\right) \wedge \mu\left(\beta_{2}, \mu(v, \tilde{\mathcal{F}})\right)\right] } \\
=[\mu(\mu(\tilde{\mathcal{F}}, v), \gamma) \wedge \mu(\mu(\tilde{\mathcal{F}}, v), \lambda), \mu(\lambda, \mu(v, \tilde{\mathcal{F}})) \wedge \mu(\gamma, \mu(v, \tilde{\mathcal{F}}))]
\end{aligned}
$$

Hence, the result follows from the fact that

$$
\begin{aligned}
& \bigvee_{\lambda, \gamma \mid \mu \beta_{1}+(1-\mu) \beta_{2}=\mu \lambda+(1-\mu) \gamma}[\mu(\mu(\tilde{\mathcal{F}}, v), \lambda) \wedge \mu(\mu(\tilde{\mathcal{F}}, v), \gamma), \mu(\lambda, \mu(\nu, \tilde{\mathcal{F}})) \\
& \wedge \mu(\gamma, \mu(\nu, \tilde{\mathcal{F}}))] \\
& \geq[\mu(\mu(\tilde{\mathcal{F}}, v), \lambda), \mu(\lambda, \mu(\nu, \tilde{\mathcal{F}}))] \wedge[\mu(\mu(\tilde{\mathcal{F}}, v), \gamma), \mu(\gamma, \mu(\nu, \tilde{\mathcal{F}}))]
\end{aligned}
$$

Theorem 8: Let $\mu^{\tilde{\mathcal{F}}} \in G F S(\mathcal{R})$. If $\mu^{\tilde{\mathcal{F}}}$ is a type 2 fuzzy convex set, then $\mu_{\left(\mu^{\mu} v+(1-\mu)^{\mu} v\right)}^{\tilde{\mathcal{F}}}$ is a generalized convex fuzzy set in $I^{\star}$ for all $v \in{ }^{+} \mu_{[0]}^{\tilde{\mathcal{F}}}$ and $\mu \in I^{\star \star}$.

Proof: It is similar to the proof of Theorem 7.

Theorem 9: If $\mu^{\tilde{\mathcal{F}}} \in G F S(\mathcal{R})$, then $\mu^{\tilde{\mathcal{F}}}$ is a type 2 fuzzy convex set if and only if $\mu_{(\mu \lambda+(1-\mu) \gamma)}^{\tilde{\mathcal{F}}}$ is a generalized convex fuzzy set in $I^{\star}$ for all $(\lambda, \gamma) \in{ }^{+} \mu_{[0]}^{\tilde{\mathcal{F}}} \times{ }^{+} \mu_{[0]}^{\tilde{\mathcal{F}}}$ and $\mu \in I^{\star \star}$.

Proof: Suppose that $\mu^{\tilde{\mathcal{F}}}$ is a type 2 fuzzy convex set and $\sigma, \pi \in I^{\star}$. Then, from the extension principle for fuzzy sets, we obtain

$$
\begin{aligned}
& \mu_{(\mu \lambda+(1-\mu) \gamma)}^{\tilde{\mathcal{F}}}(\mu \sigma+(1-\mu) \pi) \\
& \geq[\mu(\mu \odot \mu(\tilde{\mathcal{F}}, \lambda) \oplus(1-\mu) \odot \mu(\tilde{\mathcal{F}}, \gamma), \mu \sigma+(1-\mu) \pi), \mu(\mu \sigma \\
&+(1-\mu) \pi, \mu \odot \mu(\lambda, \tilde{\mathcal{F}}) \oplus(1-\mu) \odot \mu(\gamma, \tilde{\mathcal{F}}))] \\
&=\bigvee_{\theta, \alpha \mid \mu \sigma+(1-\mu) \pi=\theta+\alpha}[\mu(\mu \odot \mu(\tilde{\mathcal{F}}, \lambda), \theta) \\
& \wedge \mu((1-\mu) \odot \mu(\tilde{\mathcal{F}}, \gamma), \alpha), \mu(\theta, \mu \odot \mu(\lambda, \tilde{\mathcal{F}})) \\
&\wedge \mu(\alpha,(1-\mu) \odot \mu(\gamma, \tilde{\mathcal{F}}))]
\end{aligned}
$$




$$
\begin{aligned}
& =\bigvee_{\theta, \alpha \mid \mu \sigma+(1-\mu) \pi=\theta+\alpha}\left[\mu\left(\mu(\tilde{\mathcal{F}}, \lambda), \mu^{-1} \theta\right)\right. \\
& \wedge \mu\left(\mu(\tilde{\mathcal{F}}, \gamma),(1-\mu)^{-1} \alpha\right), \mu\left(\mu^{-1} \theta, \mu(\lambda, \tilde{\mathcal{F}})\right) \\
& \left.\wedge \mu\left((1-\mu)^{-1} \alpha, \mu(\gamma, \tilde{\mathcal{F}})\right)\right] \\
& =\bigvee_{\theta, \alpha \mid \mu \sigma+(1-\mu) \pi=\mu \theta+(1-\mu) \alpha}[\mu(\mu(\tilde{\mathcal{F}}, \lambda), \theta) \wedge \mu(\mu(\tilde{\mathcal{F}}, \gamma), \alpha), \mu(\theta, \mu(\lambda, \tilde{\mathcal{F}})) \\
& \wedge \mu(\alpha, \mu(\gamma, \tilde{\mathcal{F}}))] \\
& =\bigvee_{\sigma, \pi \mid \mu \theta+(1-\mu) \alpha=\mu \sigma+(1-\mu) \pi}[\mu(\mu(\tilde{\mathcal{F}}, \lambda), \sigma) \wedge \mu(\mu(\tilde{\mathcal{F}}, \gamma), \pi), \mu(\sigma, \mu(\lambda, \tilde{\mathcal{F}})) \\
& \wedge \mu(\pi, \mu(\gamma, \tilde{\mathcal{F}}))] \\
& \geq[\mu(\mu(\tilde{\mathcal{F}}, \lambda), \sigma) \wedge \mu(\mu(\tilde{\mathcal{F}}, \gamma), \pi), \mu(\sigma, \mu(\lambda, \tilde{\mathcal{F}})) \wedge \mu(\pi, \mu(\gamma, \tilde{\mathcal{F}}))]
\end{aligned}
$$

To prove the converse, since $\mu_{(\mu \lambda+(1-\mu) \gamma)}^{\tilde{\mathcal{F}}}$ is a generalized convex fuzzy set in $I^{\star}$, we have

$$
\begin{aligned}
\mu_{(\mu \lambda+(1-\mu) \gamma)}^{\tilde{\mathcal{F}}} & (\mu \sigma+(1-\mu) \pi) \\
& \geq[\mu(\mu(\tilde{\mathcal{F}}, \lambda), \sigma) \wedge \mu(\mu(\tilde{\mathcal{F}}, \gamma), \pi), \mu(\sigma, \mu(\lambda, \tilde{\mathcal{F}})) \wedge \mu(\pi, \mu(\gamma, \tilde{\mathcal{F}}))]
\end{aligned}
$$

for all $\sigma, \pi \in I^{\star}$. Hence,

$$
\begin{array}{r}
\mu_{(\mu \lambda+(1-\mu) \gamma)(\alpha)}^{\tilde{\mathcal{F}}} \geq \bigvee_{\sigma, \pi \mid \alpha=\mu \sigma+(1-\mu) \pi}[\mu(\mu(\tilde{\mathcal{F}}, \lambda), \sigma) \wedge \mu(\mu(\tilde{\mathcal{F}}, \gamma), \pi), \mu(\sigma, \mu(\lambda, \tilde{\mathcal{F}})) \\
\wedge \mu(\pi, \mu(\gamma, \tilde{\mathcal{F}}))] \\
=\bigvee_{\sigma, \pi \mid \alpha=\sigma+\pi}\left[\mu\left(\mu(\tilde{\mathcal{F}}, \lambda), \frac{\sigma}{\mu}\right) \wedge \mu\left(\mu(\tilde{\mathcal{F}}, \gamma), \frac{\pi}{1-\mu}\right), \mu\left(\frac{\sigma}{\mu}, \mu(\lambda, \tilde{\mathcal{F}})\right)\right. \\
\left.\wedge \mu\left(\frac{\pi}{1-\mu}, \mu(\gamma, \tilde{\mathcal{F}})\right)\right]
\end{array}
$$




$$
\begin{gathered}
=\bigvee_{\sigma, \pi \mid \alpha=\sigma+\pi}[\mu(\mu \odot \mu(\tilde{\mathcal{F}}, \lambda), \sigma) \wedge \mu((1-\mu) \odot \mu(\tilde{\mathcal{F}}, \gamma), \pi), \mu(\sigma, \mu \odot \mu(\lambda, \tilde{\mathcal{F}})) \\
\wedge \mu(\pi,(1-\mu) \odot \mu(\gamma, \tilde{\mathcal{F}}))] \\
=[\mu(\mu \odot \mu(\tilde{\mathcal{F}}, \lambda) \oplus(1-\mu) \odot \mu(\tilde{\mathcal{F}}, \gamma), \alpha), \mu(\alpha, \mu \odot \mu(\lambda, \tilde{\mathcal{F}}) \oplus(1-\mu) \\
\odot \mu(\gamma, \tilde{\mathcal{F}}))]
\end{gathered}
$$

Theorem 10: If $\mu^{\tilde{\mathcal{F}}} \in G F S(\mathcal{R})$, then $\mu^{\tilde{\mathcal{F}}}$ is a type 2 fuzzy convex set if and only if $\mu_{\left(\mu^{\mu} \lambda+(1-\mu)^{\mu} \gamma\right)}^{\tilde{\mathcal{F}}}$ is a generalized convex fuzzy set for all $\lambda, \gamma \in{ }^{+} \mu_{[0]}^{\tilde{\mathcal{F}}}$ and $\mu \in I^{\star \star}$.

Proof: It is similar to the proof of Theorem 9.

Theorem 11 [14]: Let $\mu^{\tilde{\mathcal{F}}} \in G F S(\mathcal{R})$ be a generalized semistrongly convex fuzzy set. If $+\mu_{\left[\beta_{1}, \beta_{2}\right]}^{\tilde{\mathcal{F}}}$ is closed for every $\left(\beta_{1}, \beta_{2}\right) \in I^{\star} \times I^{\star}$, then $\mu^{\tilde{\mathcal{F}}}$ is a generalized convex fuzzy set on $\mathcal{R}$.

Corollary 12: Let $\mu^{\tilde{\mathcal{F}}} \in G F S(\mathcal{R})$ and ${ }^{+} \mu_{\left[\beta_{1}, \beta_{2}\right]}^{\tilde{\mathcal{F}}}$ be closed for every $\left(\beta_{1}, \beta_{2}\right) \in I^{\star} \times$ $I^{\star}$. If $\mu_{\left(\mu^{\mu} \lambda+(1-\mu)^{\mu} \gamma\right)}^{\tilde{\mathcal{F}}}$ is a generalized semistrongly convex fuzzy set for all $\lambda, \gamma \in$ $+\mu_{[0]}^{\tilde{\mathcal{F}}}$ and $\mu \in I^{\star \star}$, then $\mu^{\tilde{\mathcal{F}}}$ is a type 2 fuzzy convex set.

Proof: It follows from Theorem 10 and Theorem 11.

Theorem 13 [14]: Let $\mu^{\tilde{\mathcal{F}}} \in G F S(\mathcal{R})$ be a generalized convex fuzzy set. If there exists $\mu \in I^{\star \star}$ for every $(\lambda \neq \gamma) \lambda, \gamma \in{ }^{+} \mu_{[0]}^{\tilde{\mathcal{F}}}$ implies that

$$
\mu_{(\mu \lambda+(1-\mu) \gamma)}^{\tilde{\mathcal{F}}}>[\mu(\tilde{\mathcal{F}}, \lambda) \wedge \mu(\tilde{\mathcal{F}}, \gamma), \mu(\lambda, \tilde{\mathcal{F}}) \wedge \mu(\gamma, \tilde{\mathcal{F}})]
$$

then $\mu^{\tilde{\mathcal{F}}}$ is a generalized strongly convex fuzzy set on $\mathcal{R}$.

Corollary 14: Let $\mu^{\tilde{\mathcal{F}}} \in G F S(\mathcal{R})$. If $\mu^{\tilde{\mathcal{F}}}$ is a type 2 fuzzy convex set and there exists $\mu \in I^{\star \star}$ for every $(\lambda \neq \gamma) \lambda, \gamma \in{ }^{+} \mu_{[0]}^{\tilde{\mathcal{F}}}$ implies that

$$
\mu_{\left(\mu^{\mu} \lambda+(1-\mu)^{\mu} \gamma\right)}^{\tilde{\mathcal{F}}}>[\mu(\tilde{\mathcal{F}}, \lambda) \wedge \mu(\tilde{\mathcal{F}}, \gamma), \mu(\lambda, \tilde{\mathcal{F}}) \wedge \mu(\gamma, \tilde{\mathcal{F}})]
$$

then $\mu_{\left(\mu^{\mu} \lambda+(1-\mu)^{\mu} \gamma\right)}^{\widetilde{\mathcal{F}}}$ is a generalized strongly convex fuzzy set. 
Proof: It follows from Theorem 10 and Theorem 13.

\section{Conclusion}

In this paper, we presented concepts of type 2 fuzzy convexities and generalized fuzzy sets, and described their characteristics. Also, the necessary and sufficient condition for which type 2 fuzzy convex sets become the other types of generalized convex fuzzy sets is illustrated. We discussed the conditions for which generalized fuzzy sets become type 2 fuzzy convex sets. In addition, some conditions for which generalized semistrongly convex fuzzy sets become a type 2 fuzzy convex set and type 2 fuzzy convex sets become a generalized strongly convex fuzzy set are presented. Furthermore, we have demonstrated that type 2 fuzzy convex sets preserved under the fuzzy intersection operation but for the fuzzy union operation need not necessarily. Our main results could be used as a foundation for further research of fuzzy convexities, such as convex and concave fuzzy soft sets and their generalizations.

\section{References}

[1] Chalco-Cano, Y., and Rojas-Medar, S-convex fuzzy processes, Computer and Mathematics with Applications, 47 (2004) 1411-1418.

[2] Chen, J., Syau, Y., and Tnig, C.-J., Convexity and semicontinuity of fuzzy sets, Fuzzy Sets and Systems, 143 (2004) 459-469.

[3] Deli, I., Convex and concave soft sets and some properties, arXiv:1307.4960v1 [math.GM], 17 July (2013).

[4] Drewniak, J., Convex and strongly convex fuzzy sets, Journal of Mathematical Analysis and Applications, 126 (1987) 292-300.

[5] Dubois, D. and Prade, H., Fuzzy Sets and Systems: Theory and Applications, Academic Press, New York (1980).

[6] Gorzalczany, B., Approximate inference with interval-valued fuzzy sets - an outline, in: proceedings of the polish symposium on interval and fuzzy mathematics, Poznan, Poland, (1983) 89-95.

[7] Hong, D.H., and Lee, S., Some algebraic properties and a distance measure for interval-valued fuzzy numbers, Information Sciences, 148 (2002) 1-10. 
[8] Kaiqi, Y.Z., A note on convex fuzzy sets, BUSEFAL, 77 (1999) 72-74.

[9] Klir, G. J., and Yuan, B., Fuzzy Sets and Fuzzy Logic: Theory and Applications, Prentice-Hall, Upper Saddle River, N.J. (1997).

[10] Lee, K. H., First Course on Fuzzy Theory and Applications, Springer Berlin Heidelberg, NY (2005).

[11] Matloka, M., A note on $\left(\Phi E_{1}, \Phi E_{2}\right)$-Convex fuzzy processes, Advances in Fuzzy Systems, 2012 (2012), 7.

[12] Peng, Z.-Y., Long, X.-J., Lin, Z., Some new properties of strongly convex fuzzy sets, Fuzzy Information and Engineering, 62 (2009) 687-693.

[13] Qiu, D., Yang, F., Shu, L., On convex fuzzy processes and their generalizations, Int. Journal of Fuzzy Systems, 12 (2010) 268-273.

[14] Sabir, P.O., Generalized semistrongly convex fuzzy sets, Journal of Zankoy Sulaimani-Part A (Pure and Applied Sciences), Vol. 18, No. 3 (2016) 259-267.

[15] Syau, Y., Closed and convex fuzzy sets, Fuzzy Sets and Systems, 110 (2000) 287-291.

[16] Syau, Y., Low, C., and $\mathrm{Wu}, \mathrm{T} .$, A note on convex fuzzy processes, Applied Mathematics Letters, 15 (2002) 193-196.

[17] Turksen, B., Interval valued fuzzy sets based on normal forms, Fuzzy Sets and Systems, 20 (1986) 191-210.

[18] Wu, S., and Cheng, W., A note on fuzzy convexity, Applied Mathematics Letters, 17 (2004) 1127-1133.

[19] Yang, X., Some properties of convex fuzzy sets, Fuzzy Sets and Systems, 7 (1995) 129-132.

[20] Yang, X.-M., and Yang, F.-M., A property on convex fuzzy sets, Fuzzy Sets and Systems, 126 (2002) 269-271.

[21] Zadeh, L. A., Fuzzy sets, Inform. \& Control 8 (1965) 338-353.

[22] Zadeh, L. A., The Concept of a Linguistic Variable and its Application to Approximate Reasoning. Memorandum ERL-M 411, Berkeley, Ca., (1973).

[23] Zadeh, L. A., The Concept of a Linguistic Variable and its Application to Approximate Reasoning (I), (II), and (III), Inf. Sci. 8 (1975) 199-249, 301357, 9 (1975) 43-80.

[24] Zimmermann, H. J., Fuzzy Set Theory and Its Applications ( $2^{\text {nd }}$ Revised Ed.), Kluwer Academic Publishers, Boston, London (1991). 